RAIRO Operations Research

RAIRO Oper. Res. 41 (2007) 35-47

DOI: $10.1051 /$ ro:2007010

\title{
OPTIMAL TIMES OF PRICE REDUCTIONS FOR AN INVENTORY MODEL WITH PARTIAL BACKORDER AND VERTICAL SHIFT DEMAND
}

\author{
Peng-Sheng You ${ }^{1}$
}

\begin{abstract}
This paper investigates an inventory control problem where a firm orders and sells an inventory item through discount strategy in a price sensitive market. From the economic points of view, customers may expect a further price reduction when a firm uses pricing promotion to stimulate demand, the demand curve may vertically shift down when a firm reduces the selling price. Taking these phenomena into account, this paper developed a continuous inventory model for finding the ordering quantity, the number of pricing changing and times of price changes simultaneously so as to maximize the total profit. A solution procedure is developed for finding the optimal decision rules.
\end{abstract}

Keywords. Inventory, backorder, deterministic demand, multiple discounts.

\section{INTRODUCTION}

In a conventional economic ordering quantity model, several conditions should be satisfied. These conditions include (1) demand is price independent, (2) backorder is not allowed, and (3) the times of price reductions are not under control, and so on. Such assumptions may not always be true. Because, in reality, demand for products such as vegetables, medicines, as well as computer hardware can evidently be affected by sale price, back-orders for products such as electric appliance and furniture are accepted by many customers, and a firm may choose a suitable time to cease a promotion plan and the regular sales price is reset after promotion

Received May 5, 2005. Accepted September 7, 2006.

1 Graduate Inst. of Transportation \& Logistics, National Chia-Yi University, 300 Shiue-Fu Road, Chia-Yi 600, Taiwan; psyuu@mail.ncyu.edu.tw

(C) EDP Sciences, ROADEF, SMAI 2007 
season. In this paper, we refer to the length between two successive time points at which the regular sales price is set as a price cycle. In a price cycle, this paper allows multiple discounts and multiple inventory replenishments. In addition, this paper consider the situation in which demands may be influenced by price and customers may expect a further price reduction when firms provide price discount.

The conventional economic ordering model thus can not be applied to this problem and need some modifications. The inventory problem has received much attention in the news vendor problem. The news vendor problem seeks to determine the stock quantity before the start of the selling period commences under probability demand. Comprehensive reviews can be found in Khouja [12] and Petruzzi and Dada [17].

Gerchak and Parlar [8] dealt with a newsboy problem in which a vendor can alter the demand distribution through costly sales effort. Gallego and Moon [6] proposed a newsboy model in which the requirement on demand information is the mean and the variance only. Moon and Choi [16] studied a newsboy problem in which customers will tend to balk at purchasing when the selection of available inventory falls below a certain threshold. Moreover, Dave, Fitzpatrick and Baker [3] investigated a deterministic production lot size inventory model in which demand is a convex function of price. Khouja [10] investigated a variety of newsboy problems in which an emergency supply can be called upon.

It is observed that the demand in the conventional news vendor literature is assumed to follow a known distribution function. This assumption implies that the pricing factors is taken as exogenous variables [20]. However, in many cases, demand frequently depends on price [9].

Thus, it is important to take into consideration the inventory problems with price dependent demand. Demand with price dependent can be roughly categorized into two cases: additive demand type and multiplicative demand type [17]. The additive demand types are such as $d(p)=\alpha-\beta p$ where $\alpha$ and $\beta$ are respectively a constant intersection and slop of the demand curve function. The multiplicative demand types are such as $d(p)=a p^{-b}$ where $a$ and $b$ are constants.

Lau and Lau [15] investigated an inventory model in which price strategy can be employed to influence demand. Polatoglu [18] developed an inventory model for determining pricing decision and procurement decisions simultaneously. Gallego and Ryzin [7] discussed the problem of optimizing the timing of selling price changes. Feng and Gallego [4] investigated the problem of determining the optimal timing of a single price change for seasonal products. Specially, the above two works considered a situation in which a single opportunity exists to change the selling price.

Feng and Xiao [5] discussed the problem of determining the times to change prices under the condition that the sales prices are predetermined. Wee [21] studied an inventory model in which demand is price dependent and inventory deteriorates at a varying rate, and proposed an algorithm for determining the maximum net profit. Khouja and Mehrez [11] provided an algorithm for solving a multi-product multi-discount constrained newsboy problem. Their model contains discount prices but not decision parameters. Later, building upon Khouja 
and Mehrez's work [11], Khouja [13] investigated a newsboy problem in which discount prices are decision variables and discount strategies are used to sell excess inventory. Urban and Baker [20] investigated a deterministic inventory problem in which demand is a multivariate function of price, time and inventory level. Their basic model with a single price is extended to a model with a single price markdown. Chiu [2] investigated a $(Q, r)$ inventory system in which each item has fixed liftime. Additionally, Chen and Chu [1] considered a related production planning problem in which demand can be controlled via pricing strategies during the selling period. Shinn and Hwang [19] dealt with the problem of determining the order quantity under the condition that demand is a convex function of price and the delay in payments is order-size dependent. Khouja [14] studied a newsboy problem with multiple discounts strategy.

Many researchers have investigated inventory models with additive price dependent demand under the condition that the intersection of demand curve is independent with retailer's pricing behavior. However, from the view point of economic phenomenon, the demand curve may vertically shift down since customers may expect a further price reduction when retailers change their selling prices. It is noted that few works have looked into inventory problems with this phenomenon.

In addition, many models have considered the back-ordering situation. However, these models have rarely simultaneously considered the situations in which demand is price dependent, partial back-order is allowed, multiple discounts are allowed, multiple replenishments are allowed and the times of replenishments are controllable. By simultaneously taking the above situations into account, this paper addresses the simultaneous determination of ordering quantity, the length of price cycle, the times of replenishments and the times of price changes.

The following sections of this paper are organized as follows. Section 1 outlines all assumptions made and formulates the problem as a mathematical model. Section 2 then analyzes the model and develops solution procedure to find decision rules. Section 3 elucidates the features of the proposed model, using a numerical example. Conclusions are finally drawn in Section 4.

\section{Assumptions And Formulation}

This paper considers the case in which a firm employees a multiple discount strategy to sell a certain inventory item. The purpose of this firm is to maximize the unit time profit. We assume that the firm sets the sales price for the item at regular sales price $p$ initially and then sequentially reduces its sales prices at a predetermined amount $r$ to influence demand. Let $k_{j}$ and $p_{j}$ be respectively the time and the sales price of the $j$ th price setting. In addition, denote the time interval between $k_{j}$ and $k_{j+1}$ as sales period $j$. The sales price during sales period $j$ is then given by $p_{j}=p-(j-1) r$. Postulate that the regular sales price $p$ is reset again immediately after the time of $k_{n+1}$. We refer to the length between $k_{1}=0$ and $k_{n+1}$ as a price cycle and the symbol $n$ be the number of price settings in a 
price cycle. It is noted that no price reduction is made if $n=1$ is set and a price cycle is composed of $n$ sales periods.

Let $d(j)$ be the demand rate during sales period $j$ and assume that it follows a linear curve of $\alpha(j)-\beta p_{j}$ where the values of $\alpha(j)$ and $\beta$ are known and constants, and respectively represent the intersection and the slop of the demand curve. In many practice, customers may expect a further price reduction when a firm uses pricing promotion to stimulate demand. Thus, we assume that the intersection of the demand curve may vertically move down when the firm reduces the sales price and is assumed to be the form of $\alpha(j)=\alpha-j \lambda$.

Assume that the firm divided a sales period into an advance sales period and a spot sales period. Each sales period starts with an advance sales period and then a spot sales period. We refer to the advance sales period of sales period $j$ as advance sales period $j$ and the spot sales period of sales period $j$ as spot sales period $j$. Assume that all demand are backordered during an advance sales period, an advance sales stops when the cumulative backorder demands reach a prespecified quantity $-s$ and all backordered demands are satisfied exactly at the ending time of an advance sales period. The arrival time of the replenishments order in sales period $j$ is assumed to be at the ending time of advance sales period $j$. The replenishment order, $Q$ is assumed to be the same for all replenishments.

The probability of customer's purchasing the item in advance is assumed to be dependent on the time length between the purchasing time and the delivery time. The delivery time is assumed to be at the ending time of an advance sales period.

Since the purchasing behavior of customers' purchasing the item in advance may be influenced by the waiting time from the time of purchase to the time of receiving their orders The probability of customer purchasing the item with backorder is assumed to be the form of $d(j) /\left(1+\delta\left(T_{j}-t\right)\right)$ where $T_{j}$ is the arrival time of a replenishment order of sales period $j, \delta$ is a positive constant and $\left(T_{j}-t\right)$ is the waiting time from the time of purchase to the time of receiving their orders.

It is noted that the time $T_{j}$ also represents the ending time of advance sales period $j$. Immediately after time $T_{j}$, we can observe that the inventory level reaches $z=Q-s$ and spot sales of sales period $j$ starts. We assume that there incur a $\operatorname{cost} c_{o}$ for each replenishment. Finally, for a sales to be profitable, we assume that the number of price settings $n$ can not exceed a prespecified number $\bar{n}$. The number $\bar{n}$ also satisfies the condition of $p_{\bar{n}}-c_{p}>0$ where $c_{p}$ is unit purchasing cost.

The firm aims to maximize its unit time profit by simultaneously determining (1) the replenishment order quantity, $Q,(2)$ the number of price settings in a price cycle, $n,(3)$ the times of replenishments, $\mathbf{T}$, and (4) the times of price settings, $\mathbf{k}$. The notation is summarized in the following.

\section{Notation:}

$$
\begin{aligned}
& Q=\text { the replenishment order quantity; } \\
& n=\text { the number of price settings in a price cycle with } n \leq \bar{n} ;
\end{aligned}
$$




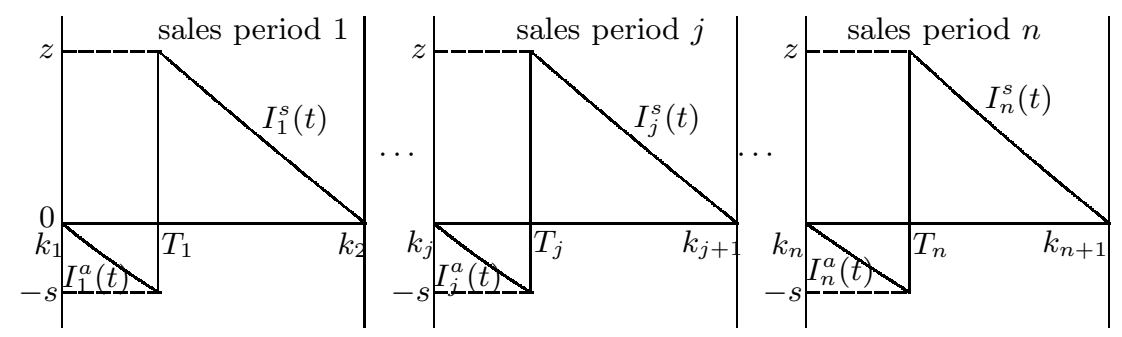

FiguRe 1. Behavior of inventory level with time in a price cycle.

$r=$ the discount amount per price reduction;

$j=$ a sales period index, sales period $j$ refers to the time interval $\left[k_{j}, k_{j+1}\right]$;

$d(j)=$ the unit time demand rate of sales period $j$ with $d(j)=\alpha-j \lambda-\beta p_{j} ;$

$p_{j}=$ the sales price of sales period $j$ with $p_{j}=p-(j-1) r$ where $p$ is the regular sales price;

$s=$ the predetermining shortage level;

$c_{h}=$ the unit inventory carrying cost, in unit time;

$c_{p}=$ the unit purchasing cost;

$c_{o}=$ the fixed ordering cost incurred with each replenishment;

$T_{j}=$ the arrival time of a replenishment order of sales period $j$;

$k_{j}=$ the starting time of sales period $j$ at which the $j$ th price setting is made;

$z=$ the maximum inventory level with $z=Q-s$.

Figure 1 depicts the changes of inventory level with time in a price cycle. Suppose the number of price settings in a cycle is set at $n$. At the start of advance sales period $j, j=1,2, \cdots, n$, there is no inventory on hand and all demands are backordered.

Let $I_{j}^{a}(t)$ denote the inventory level at time $t$ of advance sales period $j$. Since the inventory levels during this time interval decreases at time $t$ at a rate $d(j) /(1+$ $\left.\delta\left(T_{j}-t\right)\right)$, we have

$$
\frac{\mathrm{d} I_{j}^{a}(t)}{\mathrm{d} t}=\frac{-d(j)}{1+\delta\left(T_{j}-t\right)}, \quad k_{j} \leq t \leq T_{j} .
$$

At time point $T_{j}$ at which the cumulative backordered demands reach a prespecified level $-s$, the advance sales is stopped, a replenishment $Q=z+s$ arrives, all backordered demands are satisfied and the inventory level is replenished up to the level $z$. 
Let $I_{j}^{s}(t)$ denote the inventory level at time $t$ of spot sales period $j$. Then, since the inventory levels at time $t$ of this time interval decreases at a rate $d(j)=$ $\alpha-j \lambda-\beta(p-(j-1) r)$, we have

$$
\frac{\mathrm{d} I_{j}^{s}(t)}{\mathrm{d} t}=-d(j), \quad T_{j} \leq t<k_{j+1} .
$$

At time $k_{j+1}$, the $(j+1)$ th sales period starts, and the sales price is further reduced by the amount $r$ and set at $p-j r$. These process are repeated until the ending time of sales period $n, k_{n+1}$. Immediately after the ending time of sales period $n$, another price cycle starts and the sales price is set at $p$ again.

Now, we will derive the system inventory level functions $I_{j}^{a}(t)$ and $I_{j}^{s}(t)$. The inventory reaches $-s$ at the time point $T_{j}$ and the inventory level is replenished up to $z$ immediately after the time $T_{j}$. Thus, we have $I_{j}^{a}\left(T_{j}\right)=-s$ and $I_{j}^{s}\left(T_{j}\right)=z$. Using these conditions to solve differential equations (1) and (2), we have

$$
\begin{aligned}
I_{j}^{a}(t) & =\frac{\ln \left(1+\delta T_{j}-\delta t\right) d(j)}{\delta}-s, \\
I_{j}^{s}(t) & =z+\left(T_{j}-t\right) d(j) .
\end{aligned}
$$

The system inventory level is developed. Now, we will develop the unit time profit function of this problem. The profit in a price cycle is comprised of the sales revenues, inventory carrying cost, purchasing cost and ordering cost.

\section{Sales revenues:}

Let $R(n)$ be the sales revenue of a price cycle when the number of price settings is set at $n$. Then, since the sales amount in each period is $Q=z+s$ and the sales price for period $j$ is $p_{j}=p-(j-1) r$, we have

$$
R(n)=\sum_{j=1}^{n}(p-(j-1) r) Q=n(z+s)(p-0.5(n-1) r) .
$$

\section{Inventory carrying cost:}

Let $H(n)$ be the carrying cost of a price cycle when the number of price settings is set at $n$. Then,

$$
H(n)=\sum_{j=1}^{n} H_{j}(n)
$$

where $H_{j}(n)$ is the carrying cost of sales period $j$ in a price cycle when the number of price settings is set at $n$. It is noted that for sales period $j$ the inventory is positive only during spot sales period $\left[T_{j}, k_{j+1}\right]$, thus we have

$$
\begin{aligned}
H_{j}(n) & =c_{h} \int_{T_{j}}^{k_{j+1}} I_{j}^{s}(t) \mathrm{d} t \\
& =c_{h}\left(k_{j+1}-T_{j}\right) z-0.5 c_{h}\left(k_{j+1}-T_{j}\right)^{2} d(j) .
\end{aligned}
$$




\section{Purchasing cost:}

Let $C(n)$ be the total purchasing cost of a price cycle when the number of price settings is set at $n$. Then, it is clear that

$$
C(n)=n Q c_{p}=n(z+s) c_{p}
$$

\section{Ordering cost:}

Let $O(n)$ be the total ordering cost of a price cycle when the number of price settings is set at $n$. Then, we have

$$
O(n)=n c_{o}
$$

\section{Objective function}

Let $F(n, z, \mathbf{T}, \mathbf{k})$ denote the total profit of a price cycle when the number of pricing settings is set at $n$, the replenishment amount is set at $Q$, the times of price settings are set at $\mathbf{k}=\left(k_{1}, k_{2}, \cdots, k_{n}\right)$, and the times of replenishments are set at $\mathbf{T}=\left(T_{1}, T_{2}, \cdots, T_{n}\right)$. Then, from $(5) \sim(9)$ we have

$$
F(n, z, \mathbf{T}, \mathbf{k})=R(n)-H(n)-C(n)-O(n) .
$$

Since the length of a price cycle is equal to $k_{n+1}$, the unit time profit is

$$
U(n, z, \mathbf{T}, \mathbf{k})=F(n, z, \mathbf{T}, \mathbf{k}) / k_{n+1} .
$$

\section{ANALYSis}

This work aims to maximize the unit time profit by determining the number of price settings $n$, the maximum inventory level $z$, the times of price changes $\mathbf{k}$ and the times of replenishments $\mathbf{T}$. As shown in previous section, the problem is a mixed-integer nonlinear programming. It is difficult to apply the current software to solve the problem optimality. Since the objective function contains too many variables, we will reduce the number of variables by expressing unknown variables $\mathbf{T}$ and $\mathbf{k}$ in terms of $z$ and $n$. First, we will develop the following theorem.

Theorem 3.1. $T_{j}$ and $k_{j}$ has the following relationship

$$
k_{j}=T_{j}+\frac{1-\exp (s \delta / d(j))}{\delta} .
$$

Proof. Note that $I_{j}^{a}(t)=0$ for $t=k_{j}$. Thus, from (3) we obtain

$$
\frac{\ln \left(1+\delta T_{j}-\delta k_{j}\right) d(j)}{\delta}-s=0
$$

Solving the above equation produces (12). Thus, we have completed the proof. 
Since $k_{1}=0$, it is clear from (12) that

$$
T_{1}=\frac{\exp (s \delta / d(1))-1}{\delta} .
$$

For $j \geq 2$, let $\Delta T_{j}$ be the difference between $T_{j}$ and $T_{j-1}$. Then, for $j \geq 2, T_{j}$ can be found by the following theorem.

Theorem 3.2. For $n \geq 2$, we have

$$
T_{j}=\sum_{i=2}^{j} \Delta T_{i}+T_{1}
$$

where

$$
\Delta T_{j}=\frac{\exp (s \delta / d(j))}{\delta}+\frac{z}{d(j-1)}-\frac{1}{\delta} .
$$

Proof. From the fact that $I_{j}^{s}(t)=0$ for $t=k_{j+1}$, we obtain from (4)

$$
z+\left(T_{j}-k_{j+1}\right) d(j)=0
$$

from which we get

$$
k_{j+1}=T_{j}+\frac{z}{d(j)} .
$$

Combining this and (12) we have

$$
T_{j}+\frac{1-\exp (s \delta / d(j))}{\delta}=T_{j-1}+\frac{z}{d(j-1)} .
$$

Thus, we have completed the proof.

Substituting (18) into (7), we can rewrite $H_{j}(n)$ as follows

$$
H_{j}(n)=\frac{c_{h} z^{2}}{2 d(j)}
$$

Thus, we can rewrite profit function as follows:

$$
F(n, z)=n(z+s)\left(p-0.5(n-1) r-c_{p}\right)-0.5 c_{h} z^{2} \sum_{j=1}^{n}\left(\frac{1}{d(j)}\right)-n c_{o}
$$


By (14), (15) and (16), we can rewrite $k_{j+1}=T_{j}+z / d(j)$ in (18) as

$$
\begin{aligned}
k_{j+1} & =\sum_{i=2}^{j} \Delta T_{i}+T_{1}+\frac{z}{d(j)} \\
& =\sum_{i=2}^{j}\left(\frac{\exp (s \delta / d(i))}{\delta}+\frac{z}{d(i-1)}-\frac{1}{\delta}\right)+\frac{\exp (s \delta / d(1))-1}{\delta}+\frac{z}{d(j)} \\
& =-\frac{n}{\delta}+z \sum_{i=1}^{j} \frac{1}{d(i)}+\sum_{i=1}^{j} \frac{\exp (s \delta / d(i))}{\delta} .
\end{aligned}
$$

Thus, the unit time profit function can be rewritten as follows:

$$
U(n, z)=\frac{n(z+s)\left(p-0.5(n-1) r-c_{p}\right)-0.5 c_{h} z^{2} W-n c_{o}}{-n / \delta+z W+Y}
$$

where

$$
\begin{aligned}
W & =\sum_{j=1}^{n} \frac{1}{d(j)} \\
Y & =\frac{\sum_{j=1}^{n} \exp (s \delta / d(j))}{\delta} .
\end{aligned}
$$

Below, we will develop a heuristic procedure to find the decisions. For any given number of price settings $n$, let $z(n)$ be the optimal decisions to attain the optimal value of $\max _{z} U(n, z)$. That is, $U(n, z(n))=\max _{z} U(n, z)$. Then,

$$
\max _{n, z} U(n, z)=\max \{U(1, z(1)), U(2, z(2)), \cdots, U(\bar{n}, z(\bar{n}))\}
$$

Consequently, if we can find $U(n, z(n))$ for all $n \leq \bar{n}$, the optimal decision can be determined. Note for $z(n)$ to be the $z$ attaining the optimal value of $u(n, z)$, the following conditions should be satisfied: $z(n)$ is the $z$ attaining the solution of the equation $\frac{\partial U(n, z)}{\partial z}=0, \frac{\partial^{2} U(n, z)}{\partial z^{2}}<0$ and $z(n)>0$. The first derivative of $U(n, z)$ with respected to $z$ is given by

$$
\begin{aligned}
\frac{\partial U(n, z)}{\partial z}= & \frac{n\left(p-0.5(n-1) r-c_{p}\right)-c_{h} z W}{-n / \delta+z W+Y} \\
& -\frac{\left(n(z+s)\left(p-0.5(n-1) r-c_{p}\right)-0.5 c_{h} z^{2} W-n c_{o}\right) W}{(-n / \delta+z W+Y)^{2}} .
\end{aligned}
$$


The second derivative of $U(n, z)$ with respected to $z$ is given by

$$
\begin{aligned}
\frac{\partial^{2} U(n, z)}{\partial z^{2}}= & -\frac{c_{h} W}{-n / \delta+z W+Y}-\frac{2\left(n\left(p-0.5 r(n-1)-c_{p}\right)-c_{h} z W\right) W}{(-n / \delta+z W+Y)^{2}} \\
& +\frac{2\left(n(z+s)\left(p-0.5 r(n-1)-c_{p}\right)-0.5 c_{h} z^{2} W-n c_{o}\right) W^{2}}{(-n / \delta+z W+Y)^{3}} \\
= & \frac{\delta W G}{(n-z W \delta-Y \delta)^{3}}
\end{aligned}
$$

where

$$
G=-2 \delta n(n-Y \delta+\delta W s)\left(p-0.5(n-1) r-c_{p}\right)+(n-Y \delta)^{2} c_{h}+2 \delta^{2} W n c_{o} .(29)
$$

For showing that $\frac{\partial^{2} U(n, z)}{\partial z^{2}}<0$, we need the following lemmas.

Lemma 3.3. For any given $n, n-z W \delta-Y \delta<0$ for $z>0$.

Proof. Since $\sum_{j=1}^{n} \exp (s \delta / d(j))>n$, we have $n-z W \delta-Y \delta=n-z W \delta-$ $\sum_{j=1}^{n} \exp (s \delta / d(j))<-z W \delta<0$ due to $W=\sum_{j=1}^{n}(1 / d(j))>0$.

Lemma 3.4. For any given $n \leq \bar{n}, G>0$.

Proof. Since $(n-Y \delta)^{2}>0,2 \delta^{2} W n c_{o}>0$ and by modeling assumption that $p-0.5(n-1) r-c_{p}>p-(n-1) r-c_{p}>0$, it is clear that $G>0$ if $n-Y \delta+s \delta W<0$. Let $E(\delta)=n-Y \delta+s \delta W$. Then, since $\frac{d E(\delta)}{d \delta}=s \sum_{j=1}^{n}((1-\exp (s \delta / d(j))) / d(j))<0$ and $E(0)=0$, we have $E(\delta)<0$ for $\delta>0$. Thus, we have completed the proof.

Lemma 3.5. For any given $n \leq \bar{n}, z(n)>0$.

Proof. Let $z(n)$ be the $z$ attaining the solution of the equation $\frac{\partial U(n, z)}{\partial z}=0$. Then, we have

$$
z(n)=-\frac{n-Y \delta}{\delta W}+\frac{\sqrt{M}}{c_{h} \delta W}
$$

where

$$
\begin{aligned}
M= & -2 n c_{h} \delta(n-Y \delta+\delta W s)\left(p-0.5 r(n-1)-c_{p}\right) \\
& +(n-Y \delta)^{2} c_{h}^{2}+2 c_{h} \delta^{2} W n c_{o} .
\end{aligned}
$$

Since $E(\delta)=(n-Y \delta+\delta W s)<0$ and $p-0.5 r(n-1)-c_{p}>0$, we have $M \geq(n-Y \delta)^{2} c_{h}^{2}$. Thus, we have $z(n)>-\frac{n-Y \delta}{\delta W}+\frac{\sqrt{(n-Y \delta)^{2} c_{h}^{2}}}{c_{h} \delta W}=0$.

Lemma 3.6. For any given $n, \frac{\partial^{2} U(n, z)}{\partial z^{2}}<0$.

Proof. It is clear from equation (28), Lemma 3.3 and Lemma 3.4. 
TABle 1. Parameters.

\begin{tabular}{ccccccccccc}
\hline$\alpha$ & $\beta$ & $\lambda$ & $\delta$ & $r$ & $p$ & $s$ & $c_{h}$ & $c_{p}$ & $c_{o}$ & $\bar{n}$ \\
\hline 20.0 & 0.5 & 0.05 & 0.1 & 0.5 & 25 & 200 & 0.01 & 5.0 & 100 & 15 \\
\hline
\end{tabular}

According to Lemmas 3.5 and 3.6, we have for fixed $n, z(n)$ in (30) is the optimal maximum inventory level. Thus, $Q(n)=z(n)+s$ is the optimal replenishment amount. To compute $\mathbf{T}$ and $\mathbf{k}$, we first compute $\Delta T_{j}$ by (16) for all $j$. Then, we use (15) to compute $\mathbf{T}$. Finally, we compute $\mathbf{k}$ by (12). The unit time profit $U(n, z(n))$ can be obtained by (23). The optimal number of pricing settings and related decisions can be searched by the following procedure.

\section{Solution Procedure}

Step 1 Set $n=1, n^{*}=0, k_{1}^{*}=0, Q^{*}=0$ and $U^{*}=0$.

Step 2 While $n \leq \bar{n}$ do steps $3-5$.

Step 3 Calculate $z(n)$ according to (30).

Step 4 Substituting $z(n)$ into (23) to calculate $U(n, z(n))$.

Step 5 If $U(n, z(n))>U^{*}$, then

let $n^{*}=n, U^{*}=U(n, z(n)), Q^{*}=z(n)+s$,

substitute $n^{*}$ and $z^{*}$ into (16) to compute $\Delta T_{j}$, and then compute

$\mathbf{T}$ and $\mathbf{k}$ by (15) and (12), respectively, and

let $\mathbf{k}^{*}=\mathbf{k}$ and $\mathbf{T}^{*}=\mathbf{T}$.

Step 6 Output $n^{*}, U^{*}, Q^{*}, \mathbf{k}^{*}$ and $\mathbf{T}^{*}$.

\section{NumericAl EXAMPLE}

In this section, we examine one case to examine our model. The regular sales price $p$, the discount amount $r$, the demand parameters $\alpha, \lambda$ and $\beta$, the maximum back-order quantity $s$, unit time inventory carrying cost $c_{h}$, per unit purchasing cost $c_{p}$, ordering cost per replenishment $c_{o}$ and $\bar{n}$ are assumed and listed in Table 1.

By solution procedure, the values of $U(n, z(n))^{*}, \mathbf{k}^{*}, \mathbf{T}^{*}$ and $Q^{*}$ can be obtained. Table 2 shows the results of $U(n, z(n))$. We see that the profit of 111.173 is optimal for $n^{*}=9$. The value of $z(8)$ is 3674.33 from which we obtain the optimal order quantity $Q(9)=3874.33$. Substituting $n=9$ and $Q(9)=3674.33$ into (16), we can compute $\Delta T_{j}$. According to the results, we can compute compute $\mathbf{T}^{*}$ and $\mathbf{k}^{*}$ by (15) and (12), respectively. The values of $\mathbf{k}^{*}$ and $\mathbf{T}^{*}$ are shown in Table 3.

Table 3 can be illustrated as follows: the number of price settings should be set at $n^{*}=9$. The firm sets the sales price at 25 at time 0 . Then, the firm 
TABLE 2. $U(n, Q(n))$.

\begin{tabular}{cccccccccc}
\hline$n$ & $U(n, z(n))$ & $n$ & $U(n, z(n))$ & $n$ & $U(n, z(n))$ & $n$ & $U(n, z(n))$ & $n$ & $U(n, z(n))$ \\
\hline 1 & 108.642 & 4 & 110.168 & 7 & 110.987 & 10 & 111.164 & 13 & 110.753 \\
2 & 109.234 & 5 & 110.515 & 8 & 111.114 & 11 & 111.091 & 14 & 110.492 \\
3 & 109.741 & 6 & 110.788 & 9 & 111.173 & 12 & 110.953 & 15 & 110.173 \\
\hline
\end{tabular}

TABLE $3 . \mathbf{k}^{*}$ and $\mathbf{T}^{*}$.

\begin{tabular}{ccccccccccc}
\hline$j$ & 1 & 2 & 3 & 4 & 5 & 6 & 7 & 8 & 9 & 10 \\
\hline$k_{j}$ & 0.0 & 629.72 & 1236.61 & 1822.46 & 2388.85 & 2937.16 & 3468.63 & 3984.37 & 4485.37 & 4972.52 \\
$T_{j}$ & 136.52 & 756.30 & 1354.39 & 1932.41 & 2491.78 & 3033.80 & 3559.59 & 4070.19 & 4566.52 & - \\
\hline
\end{tabular}

resets its sales prices at times $629.72,1236.61,1822.46,2388.85,2937.16,3468.63$, 3984.37 and 4485.37 at $24.5,24,23.5,23,22.5,22,21.5,21$, respectively. At time 4972.52 , the firm sets its sales price at 25 again. The length of a price cycle is 4972.52. In addition, the firm receives its order quantity, $Q^{*}=3876.33$, at times $136.52,756.30,1354.39,1932.41,2491.78,3033.80,3559.59,4070.19$ and 4566.52 , respectively.

\section{Conclusion}

This paper studied the times of pricing settings and ordering problem for an inventory system under the condition that multiple discounts and back-order are allowed. Numerous inventory models have addressed the inventory problems. However, these models have rarely considered a situation in which the intersection of demand curve may move down, and the length of distinct sales periods in a price cycle and the times of price change for each sales period are controllable. Tacking these phenomena into account, this paper developed a continuous-time model to deal with this problem. The times of price changes, the times of replenishments, the replenishment amount and the price cycle are jointly derived by a simple heuristic approach studied in this paper.

Acknowledgements. The author would like to thank the National Science Council of the Republic of China for financially supporting this research under Contract No. NSC 94-2213-E-415-003-.

\section{REFERENCES}

[1] M.S. Chen and M.C. Chu, The analysis of optimal price control model in matching problem between production and sales. Asia-Pacific J. Oper. Res. 18 (2001) 131-148.

[2] H.N. Chiu, A good approximation of the inventory level in a $(Q, r)$ perishable inventory system. RAIRO Oper. Res. 33 (1999) 29-45. 
[3] D.S. Dave, K.E. Fitzpatrick and J.R. Baker, An advertising-inclusive production lot size model under continuous discount pricing. Computers and Industrial Engineering 30 (1995) $147-159$.

[4] Y. Feng and G. Gallego, Optimal stopping times for end of season sales and optimal stopping times for promotional fares. Management Science 41 (1995) 1371-1391.

[5] Y. Feng and B. Xiao, Optimal policies of yield management with multiple predetermined prices. Oper. Res. 48 (2000) 332-343.

[6] G. Gallego and I. Moon, The distribution free newsboy problem review and extensions. J. Oper. Res. Soc. 44 (1993) 825-834.

[7] G. Gallego and G.V. Ryzin, Optimal dynamic pricing of inventories with stochastic demand over finite horizons. Management Science 40 (1994) 999-1020.

[8] Y. Gerchak and M. Parlar, A single period inventory problem with partially controllable demand. Comput. Oper. Res. 14 (1987) 1-9.

[9] S. Kar, A.K. Bhunia and M. Maiti, Deterministic inventory model with two levels of storage, a linear trend in demand and a fixed time horizon. Comput. Oper. Res. 28 (2001) 1315-1331.

[10] M. Khouja, Note on the newsboy problem with an emergency supply option. J. Oper. Res. Soc. 47 (1996) 1530-1534.

[11] M. Khouja and A. Mehrez, A multi-product constrained newsboy problem with progressive multiple discounts. Comput. Industrial Engineering 30 (1996) 95-101.

[12] M. Khouja, The single-period (news-vendor) problem: literature review and suggestions for future research. Omega-Int. J. Management Science 27 (1999) 537-553.

[13] M. Khouja, Optimal ordering, discounting, and pricing in the single-period problem. Int. J. Production Economics 65 (2000) 201-216.

[14] M. Khouja, The newsboy problem under progressive multiple discounts. European J. Oper. Res. 83 (1995) 606-614.

[15] A. Lau and H. Lau, The newsboy problem with price-dependent demand distribution. IIE Transactions 20 (1988) 168-175.

[16] I. Moon and S. Choi, The distribution free newsboy problem with balking. J. Oper. Res. Soc. 46 (1995) 537-542.

[17] N.C. Petruzzi and M. Dada, Pricing and the newsvendor problem: a review with extensions. Oper. Res. 47 (1999) 183-194.

[18] L.H. Polatoglu, Optimal order quantity and pricing decisions in single-period inventory systems. Int. J. Production Economics 23 (1991) 175-185.

[19] S.W. Shinn and H. Hwang, Optimal pricing and ordering policies for retailers under ordersize-dependent delay in payments. Comput. Oper. Res. 30 (2003) 35-50.

[20] T.L. Urban and R.C. Baker, Optimal ordering and pricing policies in a single-period environment with multivariate demand and markdowns. Eur. J. Oper. Res. 103 (1997) 573-583.

[21] H.M. Wee, A replenishment policy for items with a price dependent demand and a varying rate of deterioration. Production Planning Control 8 (1997) 494-499. 